\title{
La passion du non-naturel
}

Entretien avec Brian Richardson

Sylvie Patron

\section{(2) OpenEdition}

Journals

\section{Édition électronique}

URL : http://journals.openedition.org/pratiques/5770

DOI : $10.4000 /$ pratiques. 5770

ISSN : 2425-2042

Éditeur

Centre de recherche sur les médiations (CREM)

\section{Référence électronique}

Sylvie Patron, «La passion du non-naturel », Pratiques [En ligne], 181-182 | 2019, mis en ligne le 30 juin 2019, consulté le 20 juillet 2019. URL : http://journals.openedition.org/pratiques/5770 ; DOI : 10.4000/ pratiques. 5770

Ce document a été généré automatiquement le 20 juillet 2019.

(c) Tous droits réservés 


\title{
La passion du non-naturel
}

\author{
Entretien avec Brian Richardson ${ }^{1}$
}

\section{Sylvie Patron}

1 Thomas Boutonnet : J'aimerais savoir comment vous en êtes venu à vous intéresser à ce que vous avez nommé ensuite les «récits non naturels»? Quel a été votre point de départ?

Brian Richardson ${ }^{2}$ : Je lisais Beckett et les auteurs du Nouveau roman. Ils faisaient des choses tellement intéressantes sur les narrateurs, les personnages, les intrigues, etc., que je pensais que le premier lieu vers lequel je devais me tourner était la théorie narrative. Malheureusement, il y avait un manque. Ce qu'elle faisait était très bien, mais je regardais les index encore et encore : je cherchais Beckett ou Robbe-Grillet, il n'y avait à peu près rien sur ce qu'ils faisaient, sur ces transformations étonnantes. C'est là l'origine de mon travail. J'ai bien essayé de différer pendant plusieurs années, en attendant que la théorie s'ouvre à ces auteurs. Mais cela ne s'est pas passé. J'ai dû me mettre au travail.

3 Sylvie Patron : J'ai intitulé cet entretien « La passion du non-naturel », tu diras si cela te convient. Préfères-tu vraiment les œuvres non naturelles aux autres œuvres? Où est-ce un intérêt purement professionnel, une spécialité professionnelle?

B. R. : Je suis particulièrement intéressé par les œuvres expérimentales. Mais je n'ai pas l'intention de passer encore quinze ans de ma vie à en lire. C'est une expérience intéressante, mais voilà, je l'ai faite. Et j'attends avec beaucoup d'impatience d'avoir fini les révisions d'un livre sur l'intrigue - Narrative Beginnings, Middles and Ends [Débuts, milieux et fins narratives] -, sur des questions comme la fable et le sujet, le temps, les temporalités narratives: ce sera tellement bien de pouvoir lire à nouveau d'autres œuvres. Personne ne le soupçonne, mais j'adore les romans réalistes du XIX ${ }^{e}$ siècle. Je suis impatient de revenir à la lecture de Tolstoï, et de Thackeray, et de George Eliot. Et même de Balzac!

5 Aude Herbert : Si vous deviez citer un seul livre, quel serait le livre non naturel par excellence, celui qui illustrerait le mieux votre définition du non-naturel ?

6 B. R. : Il y en a des exemples évidents. Beckett en a écrit beaucoup. Si vous prenez la Trilogie dans son ensemble, Molloy, Malone meurt, L'Innommable, c'est un merveilleux 
exemple, en particulier le dernier volume : toutes les techniques non naturelles semblent y être à l'œuvre. Il est connu en anglais non seulement comme The Unnamable [ L'Innommable], mais comme The Unreadable [L'Illisible]. Cela dit, c'est peut-être plus intéressant quand il y a une relation dialectique avec des œuvres réalistes, avec des aspects plus réalistes, comme dans Molloy... Un autre exemple paradigmatique serait Les Versets sataniques de Rushdie, qui est encore plus non naturel que Les Enfants de minuit.

7 Ioanna Kouki : J'aimerais vous interroger sur les différences et les points communs que vous avez avec votre collègue Jan Alber. Nous avons étudié ces différences dans le séminaire. Mais vous pourriez les rendre plus concrètes pour nous.

B. R. : Beaucoup de nos travaux se recoupent. Mais nous avons des définitions différentes. La sienne est très simple, très facile à mémoriser, facile à employer, mais je pense... un petit peu fausse! La mienne est plus complexe, avec plus de nuances, mais peut-être parce que la réalité elle-même est compliquée. Elle a peut-être plus de chances d'être adéquate, précisément parce qu'elle n'est pas simple. Pour Alber, tout ce qui est impossible est non naturel, toute représentation - il ne dit même pas nécessairement « fictionnelle ». Je l'ai entendu mentionner la Bible. Cela me semble une grave erreur. Il y a deux grandes catégories, avec deux types de textes vraiment différents. Pour moi, le non-naturel est une représentation clairement fictionnelle, qui présente des éléments clairement impossibles, souvent parodiques. Ce n'est pas seulement une histoire surnaturelle. Nous opérons avec des logiques différentes. Moi, j'ai cette catégorie additionnelle, au-delà du mimétique, qui reproduit des expériences familières dans notre existence de tous les jours et familières dans les récits non fictionnels, et du nonmimétique, c'est-à-dire du conventionnel, des récits non réalistes comme les contes de fées. Pour moi, l'antimimétique est plus une parodie qu'une extension des pratiques et des procédés non mimétiques.

I. K. : Alber considère ce que vous appelez le non-mimétique comme non naturel...

10 B. R. : Oui, il appelle tout « non naturel ». Certes, il ajoute des nuances : quand un procédé a été utilisé de nombreuses fois, il devient conventionalisé. Je ne dis pas «naturalisé », car cela entrainerait des discussions. Mais pour moi, ce qui est conventionalisé n'est plus entièrement non naturel. Alber n'hésite pas à dire que tous les récits non naturels attendent simplement d'être conventionalisés. Tout se passe comme si une œuvre ne pouvait être vraiment non naturelle que pendant une très courte période. J'essaie pour ma part de formuler une conception de ce qui est non naturel de façon permanente. Pour moi, le non-naturel est ce qui résiste de façon insistante et répétée à la conventionalisation. J'admets que c'est un problème difficile, mais pas insoluble. C'est une des dernières choses sur lesquelles j'ai travaillé, pour essayer de résoudre ce problème, celui de la réception non naturelle. Prenons par exemple Molloy: on sent toujours la force du non-naturel, même si on l'a lu cinquante fois, même si ce n'est plus un choc ou si on a vu réemployer des techniques de Beckett, notamment dans des œuvres françaises. Ou encore le théâtre de l'absurde : il est trop étrange, trop inhabituel, trop impossible pour être entièrement conventionalisé.

11 I. K.: Vous avez exposé les différences, quels sont maintenant les points communs que vous avez avec Alber?

12 B. R. : Nous sommes tous les deux intéressés par la théorisation des textes postmodernes présentant des impossibilités au sens d'Alber. Nous travaillons souvent sur les mêmes 
textes, ou nous mentionnons les mêmes textes. C'est la façon de les théoriser qui est différente.

13 S. P.: Que penses-tu des thèses d'Alber concernant l'histoire littéraire, à savoir : premièrement, le développement de certains genres littéraires va de pair avec la conventionalisation d'éléments non naturels; deuxièmement, les récits postmodernistes procèdent par concentration et radicalisation de textes présentant des éléments non naturels antérieurs?

B. R. : Je crois que c'est une très bonne description. On a souvent eu cela dans beaucoup d'autres formes et beaucoup d'autres genres. Ce que fait le postmodernisme, c'est exactement cela, une forme très concentrée, évidente et même flamboyante. Mais on trouve cela aussi chez Rabelais ! Si on y regarde de près, on a toute une histoire des récits non naturels. Qui commence avec Aristophane. Quant à la question de la transformation et de l'évolution littéraire, elle est passionnante dans ce contexte. Clairement, l'animal parlant remonte à Ésope, dans le monde occidental; mais on le trouve dans toutes les cultures, celles que je connais en tout cas. On a cette fusion intéressante de la tradition des histoires d'animaux avec l'avènement du réalisme chez Tolstoï, dans Kholstomer, où on voit tout à travers la perception d'un cheval. On ne peut pas le réduire simplement à une allégorie. Ce n'est pas non plus entièrement réaliste, parce que le point de vue continue après la mort du cheval, comme le fait remarquer Chklovski.

Sophie Milcent-Lawson : Selon vous, est-ce que c'est là que, concernant le statut des animaux, pourrait se faire le partage entre le non-mimétique et l'antimimétique : d'un côté, les fables ; de l'autre, Tolstoï, qui nous amène vers autre chose?

B. R.: Quelquefois, la frontière est vague, difficile à distinguer; entre les histoires d'animaux clairement conventionnelles et les récits clairement antimimétiques, il y a des cas intermédiaires. Cela ne me semble pas un problème. La question est de savoir comment nous reconnaissons... - je n'aime pas utiliser le mot "originalité ", mais c'est bien de cela qu'il s'agit. Le récit apporte une nouvelle perception, aussi bien qu'une nouvelle forme de construction. On peut utiliser l'analogie avec la parodie: la parodie marche mieux quand un trait d'un autre style n'est pas seulement exagéré mais a aussi une application très réaliste; c'est alors qu'on apprécie à quel point il est grotesque, et qu'on apprécie le pouvoir de la parodie. En ajoutant cet élément réaliste, vous atteignez un effet inattendu, un effet qu'on peut appeler « non naturel».

17 S. P.: Nous n'avons pas encore parlé des stratégies de naturalisation ou des outils de navigation d'Alber. Tu as évoqué Kholstomer et tu as dit : «On ne peut pas le réduire à une allégorie ». J'ai un peu l'impression que tu considères que toutes les stratégies d'Alber sont des façons de réduire le non-naturel.

B. R.: Je n'ose pas parler de naturalisation devant Jonathan Culler qui a écrit si brillamment sur le sujet. D'ailleurs, nous nous sommes tous éloignés de l'idée de naturalisation, qui implique autre chose. C'est plus facile et plus clair de parler de conventionalisation. Alber a une idée très large de la convention. Si trois auteurs font la même chose, cela devient une convention. J'ai une conception plus étroite. Il faut au moins deux décennies pour qu'il y ait conventionalisation. Il y a des techniques qui sont utilisées, empruntées par plusieurs auteurs et qui produisent toujours le même effet non naturel sur le lecteur. À propos des stratégies de lecture, j'ai un article qui n'est pas encore publié, je ne peux pas en parler... Disons : « Il a été dit par un important théoricien du récit non naturel... » qu'utiliser les stratégies d'Alber pour lire et naturaliser, c'est se 
débarrasser du problème. Et ce n'est pas une façon très efficace d'avoir une théorie distincte, si on montre à quel point le non-naturel est simple et facile à récupérer par d'autres approches interprétatives. Si c'est vraiment intéressant, cela ne devrait pas pouvoir être récupéré. Le danger, c'est d'avoir des lectures trop simplifiantes et réductrices. Jan Alber est un ami, nous travaillons ensemble, c'est un excellent collègue, mais certaines de ses stratégies, en particulier l'allégorisation, me semblent réductrices. Ce qu'il y a d'intéressant dans ces textes, c'est qu'ils résistent à une allégorisation facile. Les interprètes du modernisme et du postmodernisme argumentent contre certains exemples de ces lectures faciles et réductrices. Notre travail est de faire face à la complexité et à l'irréductibilité de ces œuvres.

19 S. M.-L. : Ce qui est intéressant, c'est la prise en compte que vous faites de la réception des textes non naturels. Je ne sais pas ce que vous en pensez, mais j'ai l'impression qu'on est dans une tradition herméneutique où la lecture allégorique domine. Par exemple, dès qu'il y a un animal, on va interpréter en disant « Non, non, ce n'est pas un animal, en fait ", et on va plaquer des interprétations, religieuses, etc. Il y a une résistance d'un certain lectorat à admettre que cela puisse ne pas être simplement une allégorie et être autre chose.

S. P.: Tu penses que les travaux de la narratologie non naturelle sont importants parce qu'ils nous permettent d'échapper à ces réductions allégorisantes?

S. M.-L.: Oui, à une tradition de lecture allégorisante et interprétative qui consiste à réduire l'étrangeté, réduire la nouveauté - comme si cela avait quelque chose de dérangeant, alors que tout le plaisir est là ! - pour les remettre dans les cases habituelles. S. P. : Alors, tu n'es pas albérienne, tu es richardsonienne.

B. R. : Je peux ajouter que beaucoup d'auteurs symbolistes et modernistes introduisent des éléments symboliques et allégoriques, et c'est très bien de les lire comme ça, mais il ne faut pas réduire le reste à des lectures symboliques et allégoriques, ou encore à des lectures psychologiques. On peut citer le cas de [Harold] Pinter. Il devenait fou devant toutes ces critiques différentes qui essayaient de le réduire à une seule position naturalisante : "C'est un rêve, une vision, ou une fantaisie psychologique, ou n'importe quoi ». Mais ce n'est pas cela. Ce n'est pas ce qu'il voulait faire. Il y a même un livre appelé The Pinter Problem, qui concerne toutes les différentes tentatives pour réduire Pinter.

S. P.: Peut-être que l'intérêt des stratégies de lecture d'Alber est finalement de montrer les errements de la critique lorsqu'elle est confrontée à des éléments non naturels...

B. R. : Oui !

Jonathan Culler : Je ne suis pas familier du travail d'Alber, mais un des inconvénients, me semble-t-il, est de brouiller une distinction qui me parait importante. Dans «The Babysitter " de [Robert] Coover par exemple, un merveilleux exemple de récit non naturel, on va assez loin dans la lecture avant de décider qu'on ne peut pas donner sens au récit. Les contradictions deviennent trop grandes. Ce qu'on fait alors, c'est qu'on passe à un niveau " méta » : l'histoire porte précisément sur cette impossibilité de donner sens, de résoudre les contradictions. Il est important de conserver la distinction entre le niveau " méta », qui est important pour les étudiants qui veulent écrire des essais, et les efforts expérientiels pour naturaliser, qui échouent souvent.

Martin Heide: Pourriez-vous préciser le rapport que vous entretenez avec la narratologie cognitive? 
B. R. : J'aime la théorie narrative cognitive, en particulier quand elle est bien faite. Je n'aime pas les cognitivistes lorsqu'ils en font des applications pauvres, des descriptions limitatives. C'est particulièrement évident dans la théorie du personnage. On a vu fleurir des théories du personnage très intéressantes dans les années 1960 et 1970 : différentes sortes de personnages non mimétiques étaient discutées par toutes sortes de théoriciens, notamment Barthes, «Ce sont des êtres de papier », «Ils n'ont pas de psychologie », etc. Aujourd'hui, il y a un retour en arrière. [Peter] Dixon et [Marisa] Bortolussi, David Herman, Ralf Schneider : ils traitent les personnages comme si c'étaient des personnes. Même s'ils disent parfois : « Certains personnages divergent de ce modèle humaniste ». Le problème, ce n'est pas la théorie narrative cognitive, ce sont les théoriciens. J'adorerais les voir s'emparer sérieusement de la question. Il y a des gens qui ont travaillé dans ce sens. Reuven Tsur [auteur de What is Cognitive Poetics?] se demandait : quelle est la valeur cognitive pour apprécier la poésie ? Jouer avec les métaphores... Son argument est qu'il y a un bénéfice évolutif dans le fait d'être capable de manipuler le langage de différentes façons, de reconnaitre des usages non littéraux. Mais peu de gens ont poursuivi. Il y aurait là un champ de recherche fascinant. Qu'est-ce que cela signifie de lire des récits non naturels?

29 Aude Herbert : Y a-t-il des récits ou plus généralement des textes que vous avez renoncé à étudier ou à inclure dans vos corpus?

B. R. : Non, je ne peux pas dire cela... Alber a dit que son grand regret était de ne pas pouvoir théoriser Beckett, ou une partie de l'œuvre de Beckett. Ce n'est pas un problème pour moi. Kafka est un auteur sur lequel j'aurais aimé travailler davantage. Mais je ne me sens pas vraiment frustré. Certaines œuvres sont si extrêmes qu'il n'y a presque rien à en faire, en narratologie. Par exemple, Tristan Tzara, Le Coeur à gaz. Heureusement, c'est très court!

Mariana Valderrey : Quelles sont les principales difficultés que vous avez rencontrées, dans la théorisation ou dans la réception que les gens ont faite de la théorie?

B. R. : Un des problèmes principaux, spécialement en anglais, est que les gens lisent le mot unnatural et imaginent ce qu'il pourrait signifier; ils me prêtent des positions que je n'ai pas. Cela entraine des contresens. J'en suis désolé, mais c'est trop tard pour revenir en arrière.

M. V.: Quel pourrait être pour vous l'avenir de la narratologie non naturelle ? Est-ce que vous pensez qu'elle peut s'élargir et être appliquée de manière plus internationale?

B. R. : La narratologie non naturelle est en expansion, plus que nous ne l'aurions jamais imaginé. On en trouve maintenant des applications dans la fiction graphique, par exemple. Il serait utile de travailler avec dans les études filmiques. Dans le domaine postcolonial, c'est très excitant. Dans les jeux vidéo, les cartoons. Il y a toute une série de travaux qui se préparent. 


\section{NOTES}

1. La rencontre qui est à l'origine de cet entretien a eu lieu le lundi 9 avril 2018 à l'Université Paris Diderot. Elle réunissait neuf étudiants de mon séminaire de master, «Les récits non naturels : un nouvel objet pour la narratologie »: Thomas Boutonnet, Nicolas Dorsemaine, Martin Heide, Aude Herbert, Ioanna Kouki, Jana Mrad, Luca Penge, Lucile Sonilhac et Mariana Valderrey, auxquels s'étaient joints pour l'occasion Béatrice Bloch, Jonathan Culler et Sophie Milcent-Lawson. Je remercie tout particulièrement Aude Herbert pour l'enregistrement de cette rencontre.

2. Toutes les traductions de l'anglais sont de moi.

\section{AUTEUR}

\section{SYLVIE PATRON}

Université Paris Diderot, Cérilac, F-75013 Paris, France 